\title{
A Rare Case of a Giant Hemangioendothelioma of Neck
}

\author{
Swagatam Banerjee, ${ }^{1}$ Sharmistha Chakravarty, ${ }^{2}$ Saurav Sarkar, ${ }^{3}$ Sankar Prasad Bera ${ }^{4}$
}

\begin{abstract}
Introduction:
Hemangioendothelioma is a rare variety of vascular soft tissue neoplasm, classified as a borderline tumour with diverse modality of clinical presentation. It is seen mostly at sites like the liver, lungs and bones while very few head and neck cases have been reported.

Case Report

A rare case of an unusually large tumour mass presenting as an anterolateral neck mass with extensions to right parapharyngeal space, posterior triangle and prevertebral space is presented here. The entire tumour was removed en-masse surgically. Histopathological examination confirmed the mass to be an epitheloid hemangioendothelioma, the enormous size of which in the neck makes it unique and unusual.

Kevwords:

Hemangioendothelioma, Epithelioid; Neoplasm
\end{abstract}

$\underline{\text { ABSTRACT }}$

$\mathrm{H}$ emangioendothelioma belongs to an infrequent class of vascular tumours, first described by Weiss and Enzinger in $1982 .{ }^{1}$ It is a soft tissue tumour arising from endothelial-derived spindle cells and located in the connective tissue surrounding large to medium sized blood vessels. It is categorised as an intermediate-grade neoplasm with pathological features and clinical behaviour forming a borderline between well-differentiated hemangiomas and highly malignant angiosarcomas. ${ }^{2,3}$ Among the variants, endopapillary type is common among children while epitheloid type has a predilection for adults. ${ }^{4}$

Epitheloid hemangioendothelioma is a rare variety of vascular neoplasm and is commonly seen in upper and lower extremities, liver, lungs and bones. Few cases have been reported in head and neck including oral cavity, submandibular region, parotid, thyroid

1 - Department of ENT, Medical College, Kolkata

2 - Department of ENT, ID and Beleghata Hospital, Kolkata

3 - Department of ENT, AIIMS, Bhubaneswar

4 - Department of ENT, KPC Medical College, Kolkata

\section{Corresponding author:}

Dr Swagatam Banerjee

email: drswagatam@gmail.com gland, neck, scalp, larynx and mandible amongst which submandibular space is the most commonly affected. ${ }^{3,5}$

\section{Case Report}

This is a unique case of an unusually large hemangioendothelioma in the neck of an adult female. She presented with an anterolateral neck mass extending to the posterior triangle and parapharyngeal space on right side. The grossly huge tumour which was successfully operated by an open transcervical approach weighed 453.5 grams. It is the biggest hemangioendothelioma recorded in the head and neck region. The post-operative recovery of the patient was uneventful.

A 30 year old Indian female presented with a huge neck swelling grossly measuring $14 \mathrm{~cm} \times 10 \mathrm{~cm}$, occupying the anterior neck and extending to the posterior triangle on the right side. It had gradually increased in size over the past four years. The skin over the swelling was stretched but had a smooth surface and no engorged veins or breach of skin was seen as in Figs. 1 \& 2. It was firm in consistency, non-tender, non pulsatile, fixed mass and no movement with deglutition or nerve paresis was noted.

Fine needle aspiration report from the mass showed round/spindle shaped endothelial cells with cytoplasmic 


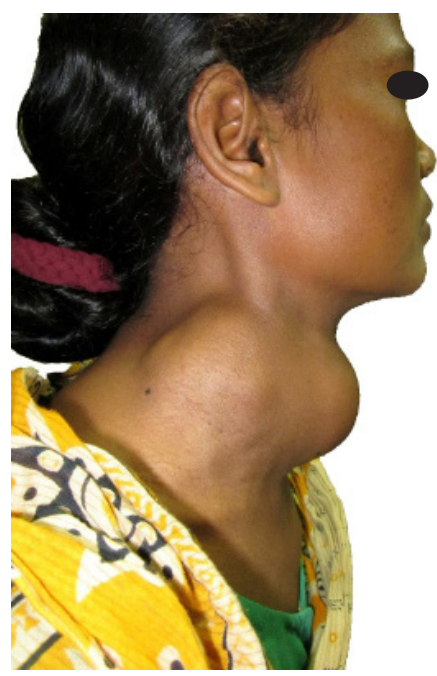

Fig. 1 Neck swelling (Right Lateral view)

vacuolation, interspersed with plump cuboidal cells. No malignant cells were found. High resolution contrast enhanced CT Scan of neck from skull base to root of neck with $5 \mathrm{~mm}$ axial cuts revealed a large homogenous, poorly enhancing hyperdense lesion, size approx. (6.1x6.3 cm) occupying the cervical region anteriorly and extending to right parapharyngeal space along with compression of great vessels on right side. Another hyperdense lesion of size approx. $(4 \times 3.5 \mathrm{~cm})$

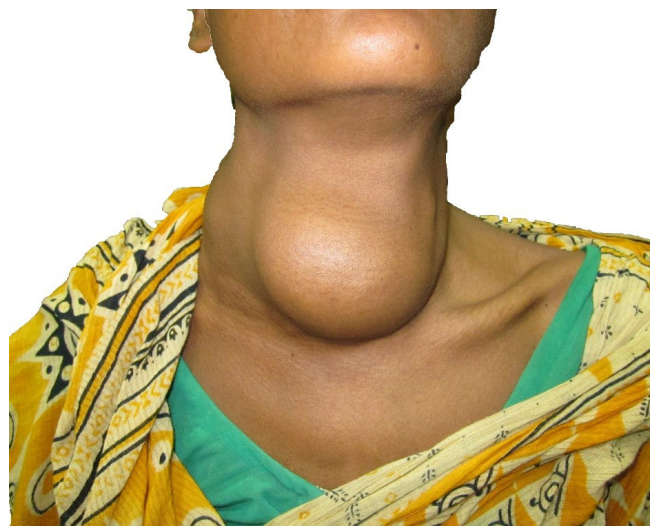

Fig. 2 Neck swelling (Anteroposterior view)

was present near the prevertebral space as in Fig 3.

USG Doppler of neck did not reveal any arterial or venous feeder to this mass despite it being a vascular tumour. An unnecessary economic burden of doing

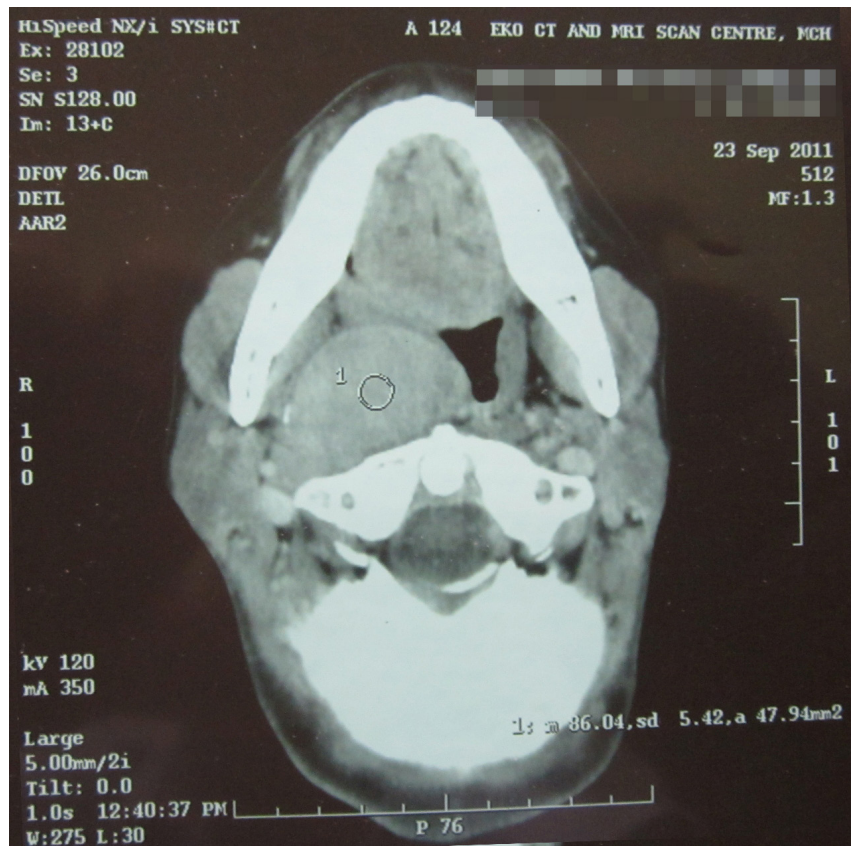

Fig. 3 CT Scan (axial cut)

a digital subtraction angiography was avoided after consultation with the interventional radiologist.

The patient was operated under general anaesthesia. Neck was extended and a horizontal skin incision was

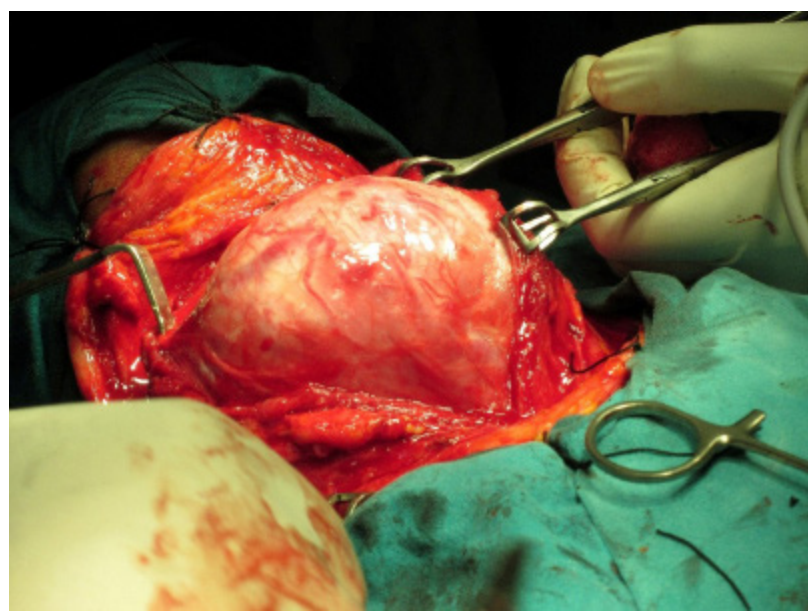

Fig. 4 Intraoperative view of neck swelling

made. The mass was exposed and was found to occupy the entire anterior aspect of neck lying just beneath the strap muscles as in Fig 4. 
Further dissection revealed its fibrous attachments to the carotid sheath, almost engulfing the sheath. Once the carotid sheath was localised, the mass was carefully dissected from the internal jugular vein to which it was particularly attached. After mobilizing the major bulk of the mass, it was found to be connected with another by a narrow band of tumour tissue in the right posterior triangle. After mobilizing the smaller mass on the right side posterior triangle and prevertebral space, the two masses along with their connection were removed en-masse from the neck as in Fig 5. There was no involvement of the nerves of the cervical region and no major intraoperative bleeding. The patient's recovery was completely uneventful post-surgery.

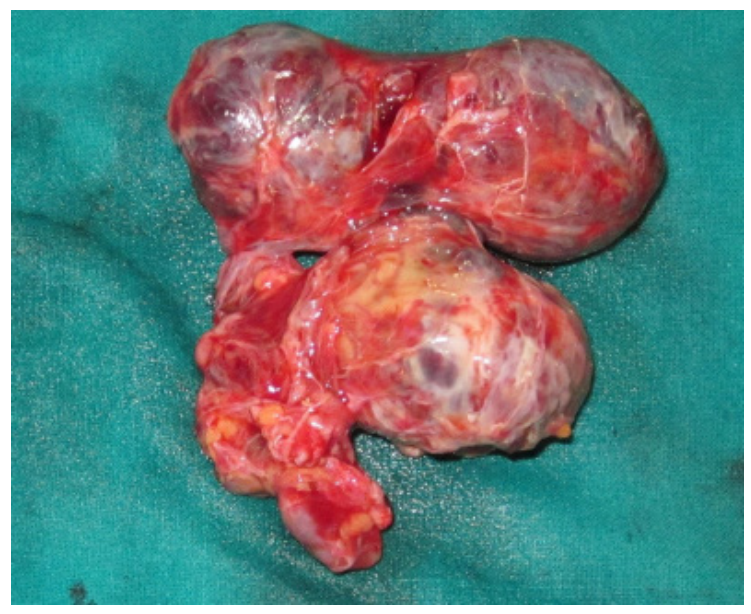

Fig. 5 Mass after surgical removal

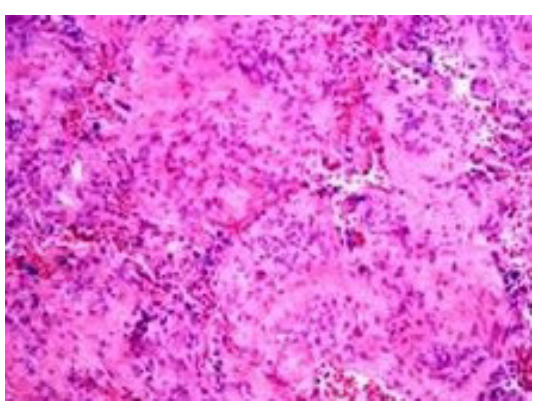

Fig. 6 Histopathological slide showing features of Hemangioendothelioma (10x, H\&E)
The histopathological report of the resected mass confirmed it as a hemangioendothelioma (Fig 6) with the discrete masses in the posterior triangle being necrotic lymphatic channels with nodes.

At the 6 months follow-up visit, she was asymptomatic and there was no clinical evidence of local recurrence or distant metastasis.

\section{Discussion}

Hemangioendothelioma in the neck is an uncommon presentation. The submandibular region in the head and neck site is most frequently involved. ${ }^{4}$ This case is unusual because of the unusually large tumour weighing 453.5 grams. Though this tumour has been reported in the head and neck region in few cases, there wasn't any existing reports of epitheloid hemangioendothelioma presenting as such a huge anterolateral neck mass. This grossly enlarged tumour, which is atypical in both size and site, and in spite of its extensive spread to posterior triangle and prevertebral space, could be completely resected after hours of meticulous dissection is probably a unique as well as unusual case.

\section{References}

1. Deyrup AT, Tighiouart M, Montag AG, Weiss SW, Epitheloid hemangioma of soft tissue: a proposal for risk stratification based on 49 cases. Am J Surg Pathol. 2008;32(6):924-927.

2. Lee JC, Lee BJ, Wang SG, Kim HW. Epitheloid hemangioendothelioma in the parapharyngeal space. J Laryngol Otol. 2006;12096):505-507.

3. Erkan AN, Bal N, Kiroglu E. A case report of hemangioendothelioma of the hard palate. Oral Surg Oral Med Oral Pathol Oral Radiol Endod. 2008 Feb;105(2):216-221

4. Mishra A, Tripathy K, Bhuyan P, Mohanty P, Rath J, Mohanty R. Endovascular papillary angioendothelioma in an elderly female. Indian J Pathol Microbiol. 2006 Jul;49(3):392-3.

5. Ellis GL, Kratochvil FJ 3rd. Epithelioid hemangioendothelioma of the head and neck: a clinicopathologic report of twelve cases. Oral Surg Oral Med Oral Pathol. 1986 Jan;61(1):61-8. 